$$
\begin{gathered}
\text { L e h r b u c h } \\
\text { der } \\
\text { Chirurgle und Operationslehre. }
\end{gathered}
$$

Vlerter Band. 



\title{
Aug. Vidal's
}

\author{
$\begin{array}{lllllllll}\mathbf{L} & \mathbf{e} & \mathbf{h} & \mathbf{r} & \mathbf{b} & \mathbf{u} & \mathbf{c} & \mathbf{h}\end{array}$ \\ der
}

\section{Chirurgie und operationslehre.}

Nach der dritten Auflage, mit besonderer Rucksicht auf das Bedürniss der Studirenden,

deutsch bearbeitet

von

\section{Dr. Adelf Iardeleben,}

opd. Professor der Chirurgie und Director der chirurgischen \#. angenărztlichen Klinik an der Universitat zu Greifswald.

Mit 533 in den Text gedruckten Holzschnitten.

Berlin, 1869.

Druck und Verlag von Georg Reimer. 
